\title{
Le rôle de la turbulence dans le fibrage du verre par le procédé à la flamme
}

\section{The role of turbulence as regards the drawing out of glass through a flame drawing process}

\author{
M. Trinité
}

\section{A. Doudou}

U.A. CNRS n 230, Faculté des sciences et des techniques
Mont Saint Aignan

Le procédé d'étirage à la flamme pour la fabrication de la laine de verre a été progressivement remplacé depuis plusieurs années par des procédés dérivés moins coûteux en énergie et à taux de production plus élevé. Toutefois, le mécanisme de base reste sensiblement le même et le travail présenté, dont l'intérêt industriel est évident, vise à expliquer le rôle de la turbulence du jet dans le mécanisme d'étirage.

The flame drawing for fiber glass production has for several years now been gradually replaced by derivative processes which are less expensive in terms of energy and result in a higher rate of production. However, the basic mechanism is roughly the same and the work presented, for which industrial interest is obvious, aims to explain the role of spraving turbulence as regards the flame drawing mechanism.

\section{Introduction}

Le procédé d'étirage à la flamme pour la fabrication de la laine de verre a été progressivement remplacé depuis plusieurs années par des procédés dérivés moins coûteux en énergie et à taux de production plus élevé. Toutefois, le mécanisme de base reste sensiblement le même et le travail présenté, dont l'intérêt industriel est évident, vise à expliquer le rôle de la turbulence du jet dans le mécanisme d'étirage.

Pour l'étirage à la flamme, on utilise un brûleur à grande vitesse de sortie appelé «brûleur jet». La combustion du mélange gazeux s'effectue presque totalement dans une chambre réfractaire de laquelle s'échappe un jet de produits de combustion très chaud $\left(2000^{\circ} \mathrm{K}\right)$ de vitesse jamais inférieure à $100 \mathrm{~m} / \mathrm{s}$.

On introduit dans ce jet une baguette de verre froid. Sous l'influence des gaz chauds, la baguette s'échauffe, se plie puis est étirée par l'action des forces aérodynamiques de traînée (fig. l).

On passe d'une fibre primaire de $0,5 \mathrm{~m}$ de diamètre à une fibre finale d'un diamètre de l'ordre de $6 \mu \mathrm{m}$ en moyenne. Le pilote qui nous a été confié par Isover comporte une seule fibre primaire. Dans le procédé industriel un grand nombre de ces fibres est alors aspiré et nappé pour former la laine de verre.

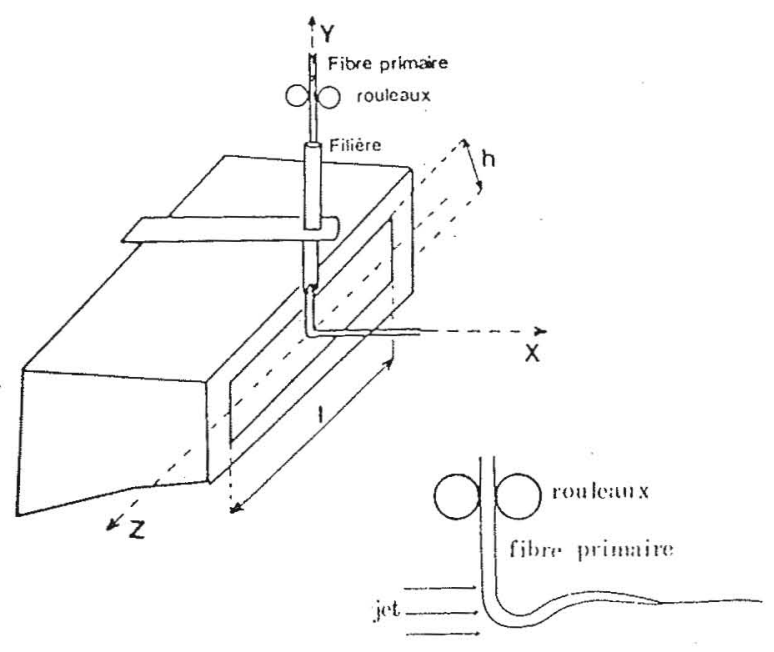

1. Schéma de principe du fibrage. 


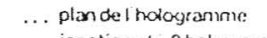

-.. - jonction de 2 hokxyram.
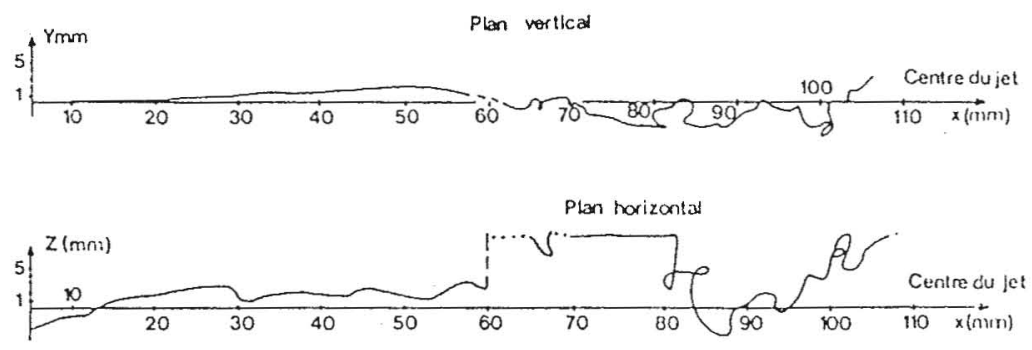

2. Exemple de trajectoire de la fibre (brileur $80 \times 10 \mathrm{~mm}$ ).

Le phénomène d'étirage ne semble pas être un phénomène simple. En effet, les visualisations holographiques que nous avions réalisées lors de travaux préalables ont montré que la fibre primaire ne donnait naissance qu'à une seule fibre secondaire. En admettant ce résultat, le calcul montre que dans les conditions normales de marche, la fibre terminale se déplace à une vitesse "débitante " de l'ordre de $600 \mathrm{~m} / \mathrm{s}$ alors que la vitesse moyenne des gaz à la sortie du brûleur n'est que de 150 $\mathrm{m} / \mathrm{s}$ environ. Le facteur multiplicatif de l'ordre de 4 a été attribué à la turbulence.

Le travail préalable (1) (2) avait consisté en la restitution spatiale de la trajectoire de la fibre en cours d'étirage et en la mesure de son diamètre (fig. 2). Par ailleurs, les mesures de turbulence par vélocimétrie laser avaient permis de caractériser la zone où il y avait interaction turbulence-fibrage.

De l'ensemble de l'étude, les hypothèses suivantes avaient été émises en guise de conclusion :

1) Après introduction de la fibre, existence d'une zone de fibrage intense où l'action des forces aérodynamiques est prépondérante d'une façon indépendante de la turbulence (la perte de diamètre est exponentielle décroissante).

Par suite de l'allongement de la fibre et de sa mise en vitesse, ces forces diminuent et deviennent très peu efficaces lorsque le diamètre atteint 10 à $20 \mu \mathrm{m}$.

2) Le diamètre final moyen de $6 \mu \mathrm{m}$ n'est atteint que grâce à l'intervention de la turbulence. Celle-ci doit être suffisamment développée et on peut émettre l'hypothèse que ce sont les fortes fluctuations accompagnées de fluide extérieur froid qui contribuent à cet étirage de façon discontinue.

L'effort devait alors porter sur l'étude directe du comportement de la fibre thermoplastique soumise à des fortes fluctuations de vitesse et de température. Pour cela nous avons proposé de mettre en œuvre l'holographie double impulsion avec une meilleure résolution et de caractériser les structures turbulentes par l'utilisation de la vélocimétrie laser associée à un traitement informatisé, ceci pour essayer de dégager les éléments en vue d'une modélisation future.

Dans la seconde partie nous présentons les caractéristiques dynamiques et thermiques du jet du point de vue de la turbulence.
La mise en œuvre de la microholographie double impulsion nous conduit, dans la troisième partie, à caractériser l'aspect turbulent de la trajectoire de la fibre.

Enfin, dans une discussion, nous essaierons de relier les échelles spatiales de la trajectoire du filament à celles temporelles de la turbulence.

La finalité à moyen terme de ce programme est l'amélioration des procédés de fabrication de la laine de verre d'une part par un accroissement du rendement d'étirage (gain d'énergie) et d'autre part par un accroissement de la qualité des fibres (recherche de la dispersion minimale au diamètre).

Le développement des méthodes de diagnostics devrait aussi pouvoir conduire à un contrôle et à une optimisation en « temps réel» de la fabrication.

\section{Caractéristiques dynamiques et thermiques du jet}

Nous nous intéresserons ici plus particulièrement aux caractéristiques turbulentes.

Les détails des champs de vitesse et de température peuvent être trouvés dans la thèse de K. Ozkul [1] et dans Verres et réfractaires [3]

\subsection{Mesure des échelles dynamiques et des spectres de vitesse}

\section{Méthode de mesure:}

La vélocimétrie Doppler à laser a été classiquement mise en œuvre en utilisant la diffusion avant (figure 3).

Le processeur (compteur TSI 1990) a une résolution de $1 \mathrm{~ns}$, ce qui permet de mesurer des fréquences Doppler allant jusqu'à $150 \mathrm{MHz}$. Ceci était nécessaire du fait des vitesses maximum rencontrées $(220 \mathrm{~m} / \mathrm{s})$ et compte tenu des caractéristiques de l'optique (espacement des faisceaux $e=50 \mathrm{~mm}$, longueur focale de la lentille $=$ $250 \mathrm{~mm}$ ). Le volume de mesure (à $1 / e^{2}$ ) avait alors un diamètre de $165 \mu \mathrm{m}$ et une longueur de $1,7 \mathrm{~mm}$.

L'ensemencement est un point important en vélocimétrie laser, en particulier si on souhaite accéder aux fré- 


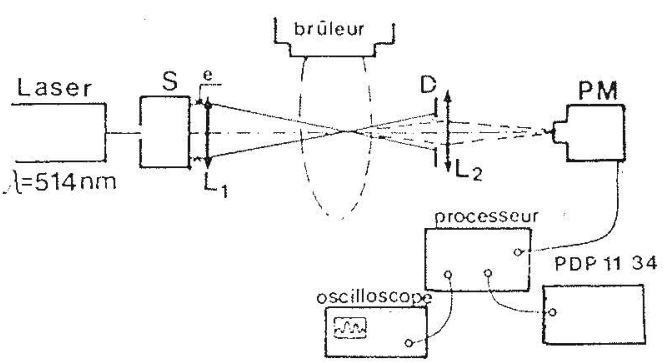

3. Dispositif de la Vélocimétrie Laser

$S$ : séparateur $\quad D$ : diaphragme $L_{2}, L_{2}$ : lentilles e: écartement PM: photomultiplicateur.

quences élevées. Nous avons utilisé une poudre réfractaire (mélange d'oxyde de magnésium $\mathrm{MGO}$ et d'oxyde de Titane $\mathrm{T}_{1} \mathrm{O}_{2}$ ) dispersée par un système brosse rotativepiston (systeme EOI). Ce dispositif nous a permis de monter à des cadences de 60000 mesures/s.

La sortie digitale du compteur est reliée à un PDP 11-34 avec accès direct mémoire (DMA).

Le programme d'acquisition permet d'accepter les mesures dont l'intervalle de temps a un minimum de $4 \mu \mathrm{s}$ $(250 \mathrm{KHz})$. Chaque mesure comprend la valeur de la vitesse de la particule et l'intervalle de temps avec la mesure précédente.

Compte tenu de l'aspect aléatoire de l'arrivée des particules, nous procédons à un rééchantillonnage à pas de temps constant* avec interpolation trapèze [4] avant d'effectuer la FFT qui nous conduit au spectre :

$$
E(v)=\int_{-\infty}^{+\infty} U^{\prime}(t) e^{-i 2 \pi v t} \mathrm{~d} t
$$

La FFT inverse nous donne la fonction d'autocorrelation

$$
R_{\mathrm{r}}=\frac{\overline{U_{1}^{\prime}, U_{1+\mathrm{r}}^{\prime}}}{\overline{U^{\prime 2}}}
$$

Par suite on peut déduire l'échelle intégrale longitudinale

$$
T_{u}=\int_{0}^{\infty} R(\tau) d \tau
$$

et la micro-échelle de Taylor $\lambda$

$$
\lambda=\lim \frac{\tau}{\sqrt{1-R(\tau)}}(\tau \rightarrow 0)
$$

\footnotetext{
* Remarques :

(1) l'échantillonage à pas de temps constant permet aussi d'éviter les biais statistiques [5].

(2) un dispositif électronique permet d'optimiser la cadence des données dans une fourchette de temps compatible avec la bande passante souhaitée de façon à ne pas encombrer inutilement le «Buffer» par des mesures trop rapprochées.
}

\section{Résultats}

Les spectres et les échelles ont été mesurés sur l'axe du jet à trois distances de la sortie du brûleur $(x=50 \mathrm{~mm}$, $100 \mathrm{~mm}$ et $150 \mathrm{~mm}$ ) la section de la buse est $L=40 \mathrm{~mm}$, $h=20 \mathrm{~mm}$.

La première station, $x / h=2,5$ caractérise le cœur potentiel du jet et les suivantes $x / h=5$ et $x / h=7,5$, le développement de la turbulence. Dans cette zone les mesures par holographie nous avaient montré qu'il y avait encore fibrage. Au-delà la température est trop basse (voir paragraphe III-2).

Dans le tableau suivant nous résumons les caractéristiques turbulentes sur l'axe de l'écoulement mesurées à ces trois stations.

\begin{tabular}{|c|c|c|c|}
\hline$X(\mathrm{~mm})$ & 50 & 100 & 150 \\
\hline$X / h$ & 2,5 & 5 & 7,5 \\
\hline $\bar{U}(\mathrm{~m} / \mathrm{s})$ & 156 & 139 & 102 \\
\hline$\left(\bar{U}^{\prime 2}\right)^{1 / 2}(\mathrm{~m} / \mathrm{s})$ & 18 & 27,2 & 28 \\
\hline$\left(\bar{U}^{\prime 2}\right)^{1 / 2} / \bar{U}(\%)$ & 11,5 & 19,5 & 27 \\
\hline$T_{u}(\mu \mathrm{s})$ & - & 170 & 245 \\
\hline$\lambda(\mu \mathrm{s})$ & - & 71 & 47 \\
\hline
\end{tabular}

Les spectres correspondants sont reportés sur les figures 4,5 et 6 (page suiv.).

Nous remarquerons qu'à la première station $(X / h=$ 2,5) l'écoulement est laminaire et dominé par une fréquence de $130 \mathrm{~Hz}$ qui doit correspondre à une résonnance acoustique du brûleur (ce qui amène le taux de «turbulence » à $11 \%$ ).

En effet, la chambre de combustion et la buse de sortie constituent un résonnateur de Helmotz dont la fréquence propre est

$$
\omega_{i}^{2}=\frac{\gamma p s}{\rho L V}
$$

$V$ volume de la chambre, $s$ section de la buse, $L$ Longueur de la buse, $p$ pression de sortie et $\gamma$ rapport des capacités calorifiques.

En considérant les caractéristiques du brûleur, nous trouvons bien une fréquence de l'ordre de $130 \mathrm{~Hz}$. Aux deux autres stations la fréquence de résonnance diminue progressivement et le spectre de turbulence s'établit suivant la pente classique en $-5 / 3$.

On remarquera que la température initiale du jet de $2000 \mathrm{~K}$ n'affecte pas l'allure du spectre.

\subsection{Caractéristiques thermiques}

Les températures ont été mesurées aux trois stations précédentes avec un thermocouple de $50 \mu \mathrm{m}$ en platine rhodié $6 \%$ - platine rhodié $30 \%$. Compte tenu des niveaux de température et de vitesse la tenue dans le temps des thermocouples était limitée. Leur temps de réponse ne 

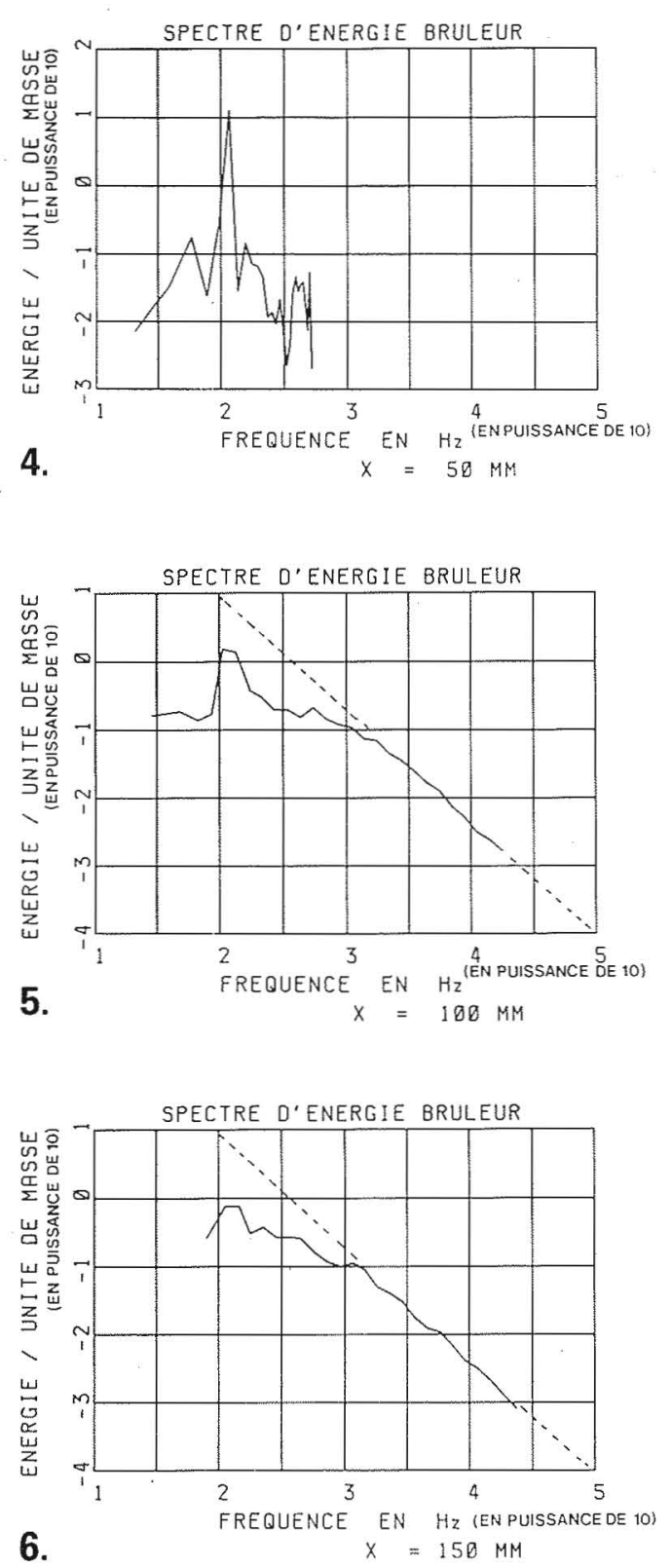

permet pas d'accéder aux fréquences des fluctuations supérieures à $250 \mathrm{~Hz}$. De plus, nous n'avons pas fait de correction de rayonnement. C'est donc une estimation de la moyenne et de l'écart type que nous montrons sur les figures 7,8 et 9 .

L'aspect dissymétrique des courbes provient de la dissymétrie de la chambre de combustion qui a été construite ainsi.

La basse fréquence acoustique qui a été mise en évidence sur les spectres dynamiques se retrouve aussi sur le signal de température. Compte tenu du temps de réponse de la sonde on ne peut rien dire sur les fluctuations hautes fréquences.

La densité de probabilité des fluctuations de température, pour les fréquences basses, montre que ces fluctuations peuvent atteindre trois fois l'écart type ce qui correspond à un écart maximum d'au moins $150^{\circ} \mathrm{C}$ entre les gaz les plus chauds et les gaz les plus froids.

Ainsi, sur l'axe du jet pour $X=150 \mathrm{~mm}$ nous déduirons $970^{\circ} \mathrm{C}<T<1120^{\circ} \mathrm{C}$. qui doit être aux environs de la limite du fibrage $\left(1200^{\circ} \mathrm{C}\right)$ si l'on tient compte de l'atténuation des hautes fréquences. Pour $X=100 \mathrm{~mm}$ nous avons $1275^{\circ} \mathrm{C}<T<1425^{\circ} \mathrm{C}$ donc toujours au-dessus de la limite du fibrage.

\section{Caractérisation de la trajectoire de la fibre par microholographie double impulsion}

Nous avions obtenu jusqu'à maintenant la restitution tridimensionnelle de la trajectoire de la fibre de verre en cours d'étirage. Il s'agissait de vues figées à un instant donné. Bien que des caméras holographiques expérimentales aient déjà été réalisées, la technique actuelle ne permet pas de suivre dans le temps des objets en déplacement rapide tels que ceux que nous voulons étudier (de l'ordre de $160 \mathrm{~m} / \mathrm{s}$ ). Une solution intéressante est l'utilisation du laser double impulsion [6] qui permet de fournir une information sur la dynamique des phénomènes.

\subsection{Dispositif expérimental}

\section{Montage d'enregistrement (fig. 10)}

C'est un montage à simple faisceau ou montage de Gabor. Le champ de mesure $\left(25 \times 25 \mathrm{~mm}^{2}\right)$ est reporté au voisinage de la plaque holographique à l'aide d'un objectif $(\mathrm{Ob})$ de focale $150 \mathrm{~mm}$. L'énergie des impulsions est contrôlée par un oscilloscope analogique à mémoire ( $f i^{-}$ gure 10).

\section{La source}

Un laser à rubis délivre deux impulsions rapprochées entre 10 et $300 \mu$ s à l'intérieur d'une seule excitation du rubis.

Le système optique se déplace en aval du brûleur (axe $\mathrm{X}$ ) pour explorer le trajet de la fibre jusqu'à $150 \mathrm{~mm}$. La résolution spatiale a été testée à l'aide d'une fente réglable de 5 à $100 \mu \mathrm{m}$ [7]. A peine ouverture $(f / 3)$, le seuil de mesure est de $5 \mu \mathrm{m}$ avec une sensibilité de $2,5 \mu \mathrm{m}$.

\section{Restitution des images}

On utilise un système de projection directe sur une table de mesure (Tm) Digiplan schématisé sur la figure 11. Avec un grandissement de l'ordre de 70, l'enregistrement de fils calibrés entre 5 et $50 \mu \mathrm{m}$ donne une précision de $+3 \mu \mathrm{m}$ dans l'espace objet (plan xoy) dans le cas d'hologramme à une impulsion. En profondeur (axe $z$ ) la précision de mise au point est de 50 à $100 \mu \mathrm{m}$ suivant les zones de mesure.

La plaque holographique est montée sur une platine motorisée à 3 degrés de liberté en translation, de sorte que les caractéristiques optiques du montage restent identiques quelque soit le point de mesure sur le trajet de la fibre.

Pour chaque hologramme, le trajet de la fibre est suivi dans les trois directions et reproduit manuellement sur les figures fournies en deux plans (xoy) et (zoy). 

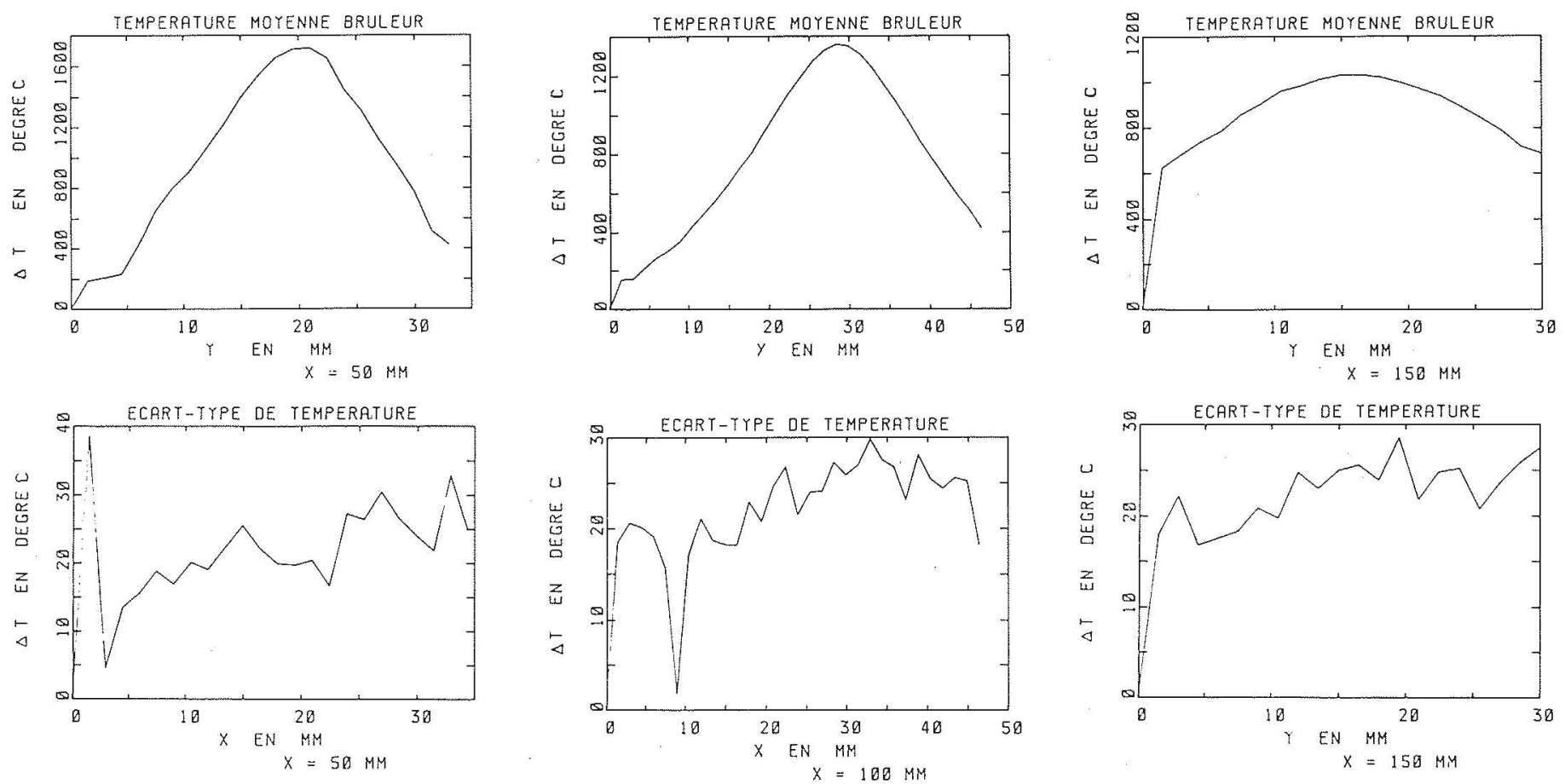

7.

8.

9.
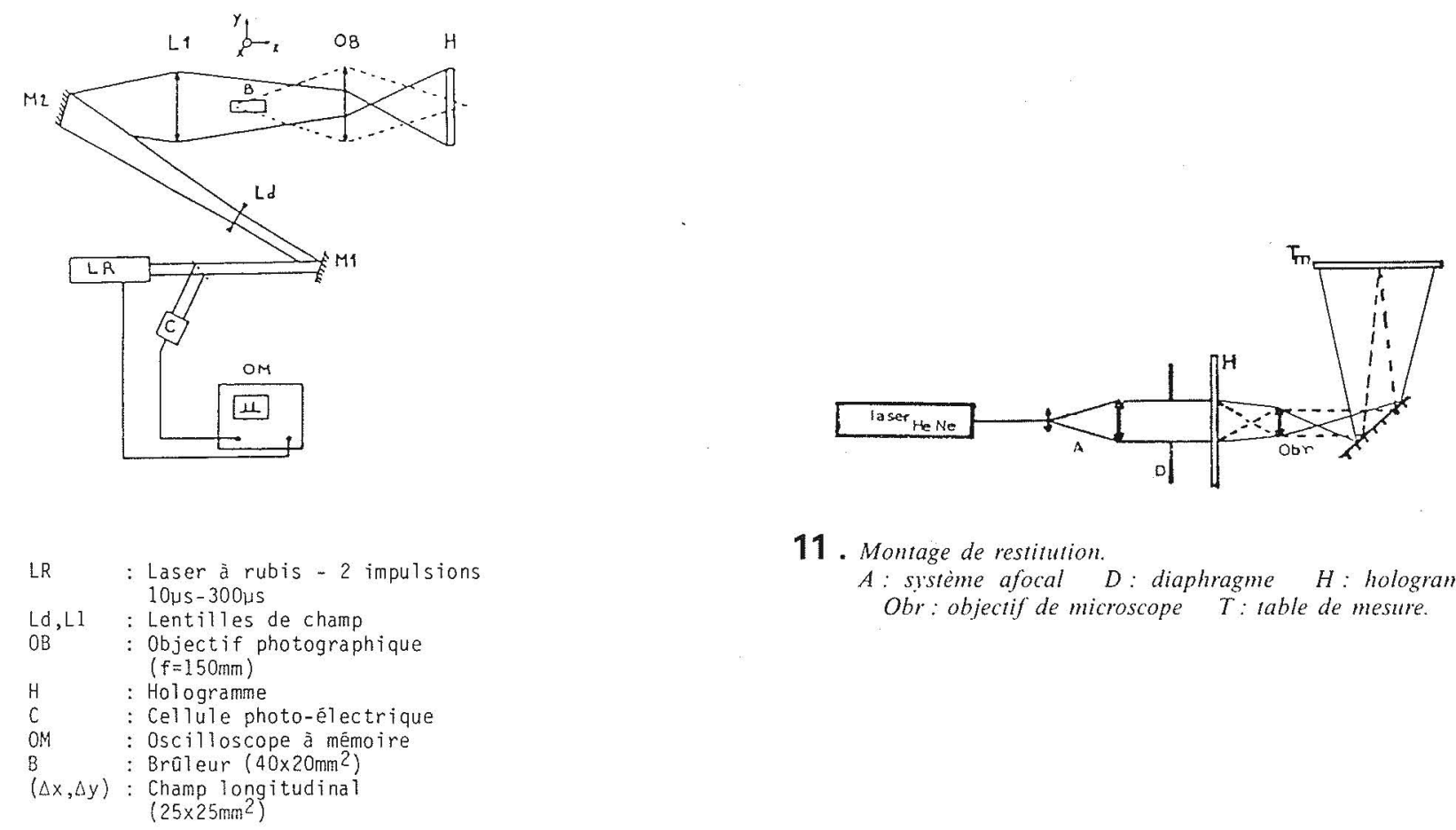

11. Montage de restitution.

A: systeme afocal $D$ : diaphragme $H$ : hologramme Obr: objectif de microscope $T$ : table de mesure.

10. Montage d'enregistrement d'hologrammes à deux impulsions. 


\section{Qualité des images en double impulsion}

La qualité de l'image est liée au contraste des franges de diffraction enregistrées sur la plaque holographique. Le signal à enregistrer se compose d'une porteuse et d'une modulation: $S=V+\Delta V$.

Au niveau de la plaque, il y a accumulation de la lumination $\left(L_{i}=E_{i} \cdot \Delta t\right)$ de chaque impulsion laser suivant la courbe de noircissement de la plaque (figure 12), en notant $V_{1}$ et $V_{2}$ l'amplitude des signaux correspondant aux ondes porteuses des 2 impulsions et $\Delta V_{1}$ et $\Delta V_{2}$ les signaux objets correspondants.

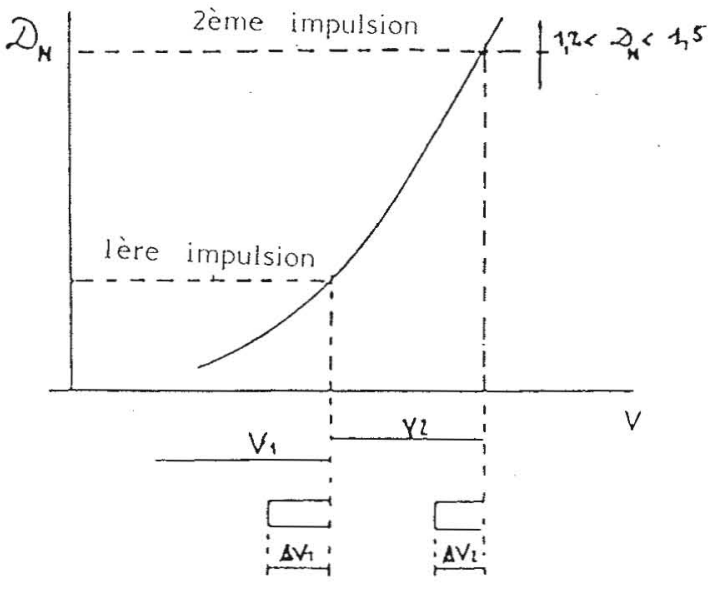

Holographie de Gubor

12. Noircissement de la plaque.

En simple exposition, le contraste de l'objet est $\Delta V / V$ et en double exposition, pour 2 images décalées dans l'espace, le contraste est affaibli : $\Delta V_{i} / V_{1}+V_{2} \quad i=1,2$.

Pour une valeur minimale de $V$ dépassant le seuil de noircissement, on réalise des hologrammes exploitables malgré la baisse de contraste, pour un écart maximal entre les impulsions $\left(V_{1}-V_{2}\right) /\left(V_{1}+V_{2}\right)$ inférieur à $10 \%$, et avec un niveau moyen $\left(V_{1}+V_{2}\right)$ dans la zone linéaire de $D_{N}=$ $f(V)\left(0,05<D_{\mathrm{s}}<0,1\right)$

\subsection{Résultats}

Les conditions générales de prise de vue ainsi que les caractéristiques de l'écoulement sont reportées dans [3]. Nous présentons une visualisation typique de la fibre en cours d'étirage pour différents retards entre les deux impulsions: 10, 24, 50 et $100 \mu \mathrm{s}$ (figures 13 à 16). Les mesures ont été faites dans la zone du jet présentant le maximum de turbulence c'est-à-dire pour $y>80 \mathrm{~mm}$. Les résultats sont présentés sous forme de deux courbes pour chaque retard: la trajectoire dans le plan $y, x$ et dans le plan $y, z$.

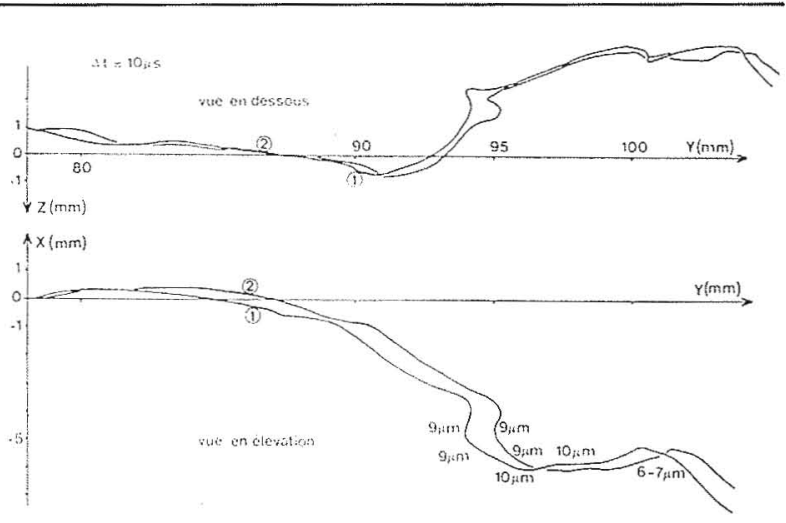

13.

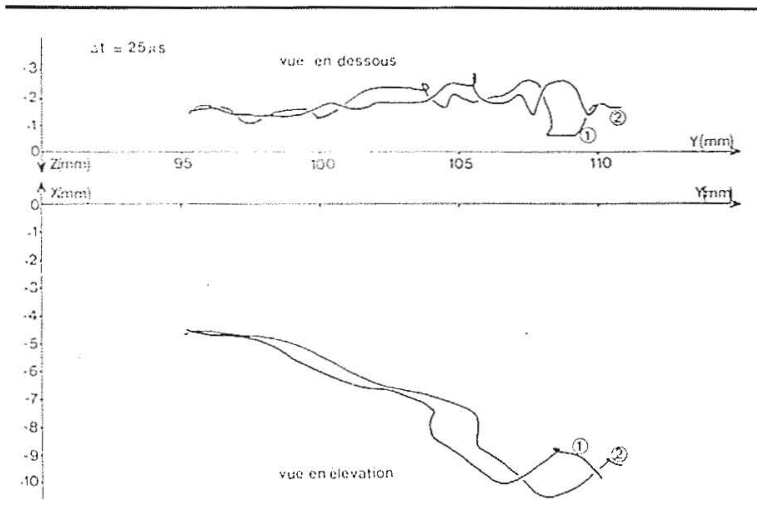

14.

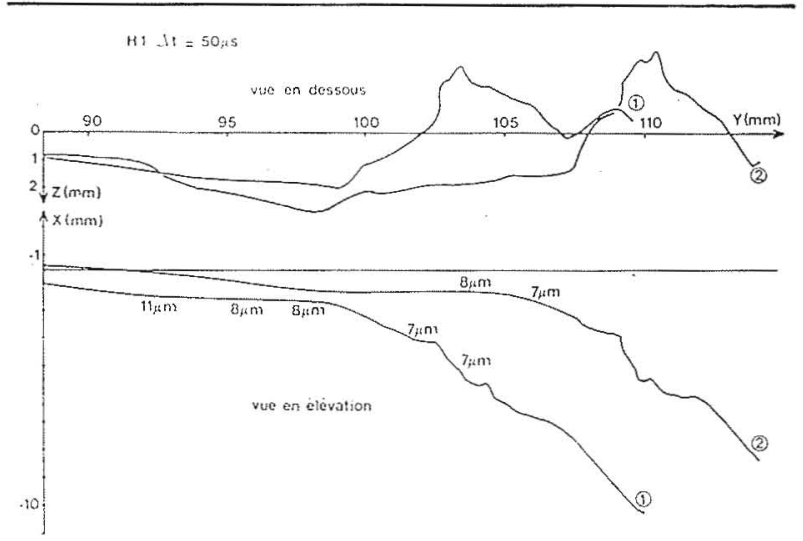

15.

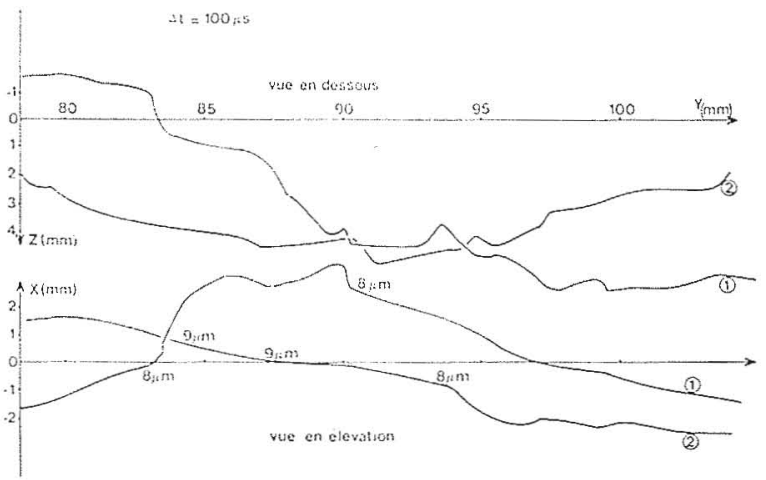

16. 
Pour suivre l'évolution de l'étirage entre les deux instants de la prise de mesure il y a lieu de repérer dans l'un ou l'autre des plans, un point caractéristique de la fibre pour accéder à la vitesse d'ensemble, et deux points caractéristiques pour accéder à l'allongement.

L'étude des courbes nous conduit aux remarques qui vont suivre :

Vitesse locale de la fibre: On retrouve des vitesses locales de la fibre de l'ordre de grandeur de la vitesse moyenne de l'écoulement avec des fluctuations importantes.

Echelle spatiale du phénomène: Pour $\Delta t=10$ us, on observe une translation de l'ensemble de la fibre sans modification notable de la courbure.

Pour $\Delta t=25 u$ s et $50 u s$, on a à la fois la translation d'ensemble et l'apparition de petites structures.
Pour $\Delta t=100 \mu \mathrm{s}$ les deux trajectoires sont pratiquement décorrélées.

Allongement de la fibre: La mesure de la longueur de la fibre ne fait pas apparaître un facteur multiplicatif plus grand que 1,5 à 1,8 . Les relevés de diamètre montrent bien le caractère local de l'allongement.

\section{Remarque concernant l'exploitation}

On retrouve ici le problème de l'exploitation des clichés holographiques. L'exploitation semi-manuelle est un travail long et fastidieux alors que tous les résultats pour avoir une valeur statistique supposeraient le traitement d'un grand nombre d'images.

L'automatisation à partir de la plaque holographique est très difficile, par contre, nous avons engagé une recherche sur l'exploitation directe de la figure de diffraction à partir d'une barrette de photodiodes [8].

\section{Discussion}

Des résultats qui ont été présentés deux points peuvent être notés :

\section{La zone de fibrage final}

On peut admettre que le fibrage aérodynamique par force de trainée s'arrête quand la vitesse de la fibre atteind la vitesse du jet, or ceci correspond à un diamètre de la fibre de 20 à $30 \mu \mathrm{m}$. Les hologrammes double impulsion nous montrent que la vitesse de la fibre a rejoint la vitesse du jet dans la zone de développement de la turbulence $(x / d$ $>5$ ). C'est donc avec l'arrivée de la turbulence que le passage à $6 \mu \mathrm{m}$ doit s'effectuer. Nous avons vu que le niveau thermique du jet est suffisant pour que cela soit possible, au moins par intermittence.

\section{Les échelles du phénomène}

La visualisation nous montre l'apparition de petites structures pour un $\Delta t$ de 25 à $50 \mu \mathrm{s}$, ceci est tout à fait en accord avec les microéchelles temporelles que nous avons mesurées (40 à $70 \mu \mathrm{s})$. En suivant l'hypothèse de Taylor, cela correspond à des échelles spatiales de l'ordre de 4 à $7 \mathrm{~mm}$.
Nous remarquons que sur les visualisations réalisées le diamètre mesuré est assez proche du diamètre final (7 à $11 \mu \mathrm{m})$.

De ces deux points nous concluons que le passage au diamètre final de la fibre ne peut se faire que lorsque par l'action des microstructures de la turbulence, le fibrage étant terminé lorsque la trajectoire de la fibre épouse parfaitement les filets fluides instantanés.

L'augmentation de la vitesse débitante d'un facteur 4 ne peut donc s'expliquer que par ce phénomène.

En admettant ce processus physique, il devrait maintenant être possible de modéliser ce phénomène, au moins pour prévoir le diamètre final minimum possible en fonction de la microéchelle du jet.

Pour être plus complètes les visualisations auraient dû être aussi réalisées dans la zone $x=50$ à $100 \mathrm{~mm}$ où le diamètre de la fibre est passé de 20 à $10 \mu \mathrm{m}$. De toute façon nous nous heurtons au problème d'exploitation de la plaque holographique qui ne permet pas de réaliser des statistiques sur un grand nombre de fibres. Afin de résoudre ce problème nous avons mis au point un système de mesure quasitemps réel de diamètre et position de la fibre basé sur l'exploitation de la figure de diffraction [8].

Pour le moment, les fluctuations de position mesurables $( \pm 1 \mathrm{~mm})$ ne sont pas encore suffisantes pour cette application. 


\section{Références}

[1] Ozkul C. - Microholographie : application à la visualisation d'une fibre en cours d'étirage. Thèse de Docteur Ingénieur, Université de Rouen (1980).

[2] Ozkul C., Allano D., Lisiecki D., Trinite M. - Visualisation holographique du fibrage de verre et mesure de hurbulence dans le jet. Rapport de fin de contrat Saint-Gobain -- ISOVER (Octobre 1980).

[3] Ozkul C., Trinite M., Allano D., Lisiecki D. - Contribution à l'étude du fibrage par le procédé à la flamme. Revue Verres et réfractaires, vol. $38, \mathrm{n}^{\circ} 2$ (Mars-Avril 1984).

[4] Doudou A. - Mesure des spectres à partir de la vélocimétrie Laser. Rapport D.E.A. (Sept. 1986).

[5] Labbaci K., Trinite M., Stepowski D. - Comparaison des différentes moyennes réalisées sur les mesures de vitesses dans une flamme de diffusion turbulente. L.D.A. Specialists Meeting, Saint-Louis, May 18-20 (1987).

[6] Trinite M., Vannobel F., Allano D. - Visualisation double impulsion dans les écoulements - Application à la Microholographie et à l'Ombroscopie. Colloque de Visualisation, Nancy, (Janvier 1985).

[7] Rapport confidentiel ISOVER-CORIA (Mai 1983).

[8] Ozkul C., Allano D., Trinite M., Anthore N. - In line far field holography and diffraction pattern analysis: new developments. SPIE, vol. 600, Progress in Hologrpahic Applications (1985). 